\title{
Series Representations at Special Values of Generalized Hurwitz-Lerch Zeta Function
}

\author{
S. Gaboury ${ }^{1}$ and A. Bayad ${ }^{2}$ \\ ${ }^{1}$ Department of Mathematics and Computer Science, University of Quebec at Chicoutimi, Chicoutimi, QC, Canada G7H 2B1 \\ ${ }^{2}$ Département de Mathématiques, Université d'Évry Val-d'Essonne, 23 Boulevard de France, 91037 Évry Cedex, France
}

Correspondence should be addressed to S. Gaboury; slgabour@uqac.ca

Received 30 August 2013; Accepted 5 November 2013

Academic Editor: Junesang Choi

Copyright (c) 2013 S. Gaboury and A. Bayad. This is an open access article distributed under the Creative Commons Attribution License, which permits unrestricted use, distribution, and reproduction in any medium, provided the original work is properly cited.

By making use of some explicit relationships between the Apostol-Bernoulli, Apostol-Euler, Apostol-Genocchi, and ApostolFrobenius-Euler polynomials of higher order and the generalized Hurwitz-Lerch zeta function as well as a new expansion formula for the generalized Hurwitz-Lerch zeta function obtained recently by Gaboury and Bayad, in this paper we present some series representations for these polynomials at rational arguments. These results provide extensions of those obtained by Apostol (1951) and by Srivastava (2000).

\section{Introduction}

The generalized Hurwitz zeta function $\zeta(s, a)$ is defined by [1, page 88 et seq.]

$$
\begin{gathered}
\zeta(s, a):=\sum_{n=0}^{\infty} \frac{1}{(n+a)^{s}} \\
\left(\operatorname{Re}(s)>1 ; a \in \mathbb{C} \backslash \mathbb{Z}_{0}^{-} ; \mathbb{Z}_{0}^{-}:=\{0,-1,-2, \ldots\}\right),
\end{gathered}
$$

where

$$
\zeta(s, 1)=\zeta(s)=\frac{1}{2^{s}-1} \zeta\left(s, \frac{1}{2}\right)
$$

yields the celebrated Riemann zeta function $\zeta(s)$. The Riemann zeta function is continued meromorphically to the whole complex $s$-plane except for a simple pole at $s=1$ with residue 1 .

The Hurwitz-Lerch zeta function $\Phi(z, s, a)$ is defined, as in [1, page 121 et seq.], by

$$
\Phi(z, s, a):=\sum_{n=0}^{\infty} \frac{z^{n}}{(n+a)^{s}}
$$

$\left(a \in \mathbb{C} \backslash \mathbb{Z}_{0}^{-} ; s \in \mathbb{C}\right.$ when $|z|<1 ; \operatorname{Re}(s)>1$ when $\left.|z|=1\right)$.
Clearly, we have the following relations:

$$
\Phi(1, s, a)=\zeta(s, a), \quad \Phi(1, s, 1)=\zeta(s) .
$$

The Hurwitz-Lerch zeta function has the well-known integral representation

$$
\Phi(z, s, a)=\frac{1}{\Gamma(s)} \int_{0}^{\infty} \frac{t^{s-1} e^{-a t}}{1-z e^{-t}} d t
$$

$$
(\operatorname{Re}(a)>0 ; \operatorname{Re}(s)>0 \text { when }|z| \leq 1(z \neq 1) ;
$$

$$
\operatorname{Re}(s)>1 \text { when } z=1) \text {. }
$$

Recently, Lin and Srivastava [2] investigated a more general family of Hurwitz-Lerch zeta functions. Explicitly, they introduced the function $\Phi_{\mu, \nu}^{(\rho, \sigma)}(z, s, a)$ defined by

$$
\begin{aligned}
& \Phi_{\mu, \nu}^{(\rho, \sigma)}(z, s, a):=\sum_{n=0}^{\infty} \frac{(\mu)_{\rho n}}{(\nu)_{\sigma n}} \frac{z^{n}}{(a+n)^{s}} \\
& \left(\mu \in \mathbb{C} ; a, \nu \in \mathbb{C} \backslash \mathbb{Z}_{0}^{-} ; \rho, \sigma \in \mathbb{R}^{+} ;\right. \\
& \rho<\sigma \text { when } s, z \in \mathbb{C} ; \\
& \rho=\sigma, s \in \mathbb{C} \text { when }|z|<1 ; \rho=\sigma, \\
& \operatorname{Re}(s-\mu+\nu)>1 \text { when }|z|=1),
\end{aligned}
$$


where $(\lambda)_{\kappa}$ denotes the Pochhammer symbol defined, in terms of the Gamma function, by

$$
\begin{aligned}
(\lambda)_{\mathcal{K}}: & =\frac{\Gamma(\lambda+\kappa)}{\Gamma(\lambda)} \\
& = \begin{cases}\lambda(\lambda+1) \cdots(\lambda+\kappa-1), & (\kappa \in \mathbb{N} ; \lambda \in \mathbb{C}) \\
1, & (\kappa=0 ; \lambda \in \mathbb{C} \backslash\{0\}) .\end{cases}
\end{aligned}
$$

It is easily seen that

$$
\begin{gathered}
\Phi_{\nu, \nu}^{(\sigma, \sigma)}(z, s, a)=\Phi_{\mu, \nu}^{(0,0)}(z, s, a)=\Phi(z, s, a), \\
\Phi_{\mu, 1}^{(1,1)}(z, s, a)=\Phi_{\mu}^{*}(z, s, a):=\sum_{n=0}^{\infty} \frac{(\mu)_{n}}{n !} \frac{z^{n}}{(n+a)^{s}} .
\end{gathered}
$$

The function $\Phi_{\mu}^{*}(z, s, a)$ is, in fact, a generalized HurwithLerch zeta function investigated by Goyal and Laddha [3, page 100, (1.5)].

Let us recall some other important special cases of the Hurwitz-Lerch zeta function $\Phi(z, s, a)$. The Lerch zeta function defined by

$$
l_{s}(\xi):=\sum_{n=1}^{\infty} \frac{e^{2 n \pi i \xi}}{n^{s}} \quad(\xi \in \mathbb{R} ; \operatorname{Re}(s)>1)
$$

is related to the Hurwitz-Lerch zeta function by the following relation:

$$
l_{s}(\xi)=e^{2 \pi i \xi} \Phi\left(e^{2 \pi i \xi}, s, 1\right)
$$

Also, we note, as a special case of the Hurwitz-Lerch zeta functions, the Lipschitz-Lerch zeta function [1, page 122, Equation (2.5)(11)]:

$$
\begin{aligned}
& \phi(\xi, a, s):=\sum_{n=0}^{\infty} \frac{e^{2 n \pi i \xi}}{(n+a)^{s}}=\Phi\left(e^{2 \pi i \xi}, s, a\right) \\
& \left(a \in \mathbb{C} \backslash \mathbb{Z}_{0}^{-} ; \operatorname{Re}(s)>0 \text { when } \xi \in \mathbb{R} \backslash \mathbb{Z} ;\right. \\
& \operatorname{Re}(s)>1 \text { when } \xi \in \mathbb{Z}) .
\end{aligned}
$$

Setting $z=\exp (2 \pi i p / q)$ with $p \in \mathbb{Z}$ and $q \in \mathbb{N}$ and using the next series identity

$$
\sum_{n=0}^{\infty} f(n)=\sum_{j=0}^{k-1} \sum_{n=0}^{\infty} f(k n+j)
$$

we obtain the following summation formula for the LipschitzLerch zeta function $\phi(\xi, a, s)$ :

$$
\phi\left(\frac{p}{q}, a, s\right)=q^{-s} \sum_{j=1}^{q} \zeta\left(s, \frac{a+j-1}{q}\right) \exp \left(\frac{2(j-1) p \pi i}{q}\right)
$$

in terms of the Hurwitz zeta function $\zeta(s, a)$ defined by (1) (see also [4, page 81, Equation (3.9)]).

Finally, we recall Lerch's functional equation [5, page 29, Equation (1.11)(7)]:

$$
\begin{aligned}
& \Phi(z, s, a)= i z^{-a}(2 \pi)^{s-1} \Gamma(1-s) \\
& \times\left\{\exp \left(-\frac{1}{2} s \pi i\right) \Phi\left(e^{-2 \pi i a}, 1-s, \frac{\log z}{2 \pi i}\right)\right. \\
&-\exp \left[\left(2 a+\frac{1}{2} s\right) \pi i\right] \\
&\left.\times \Phi\left(e^{2 \pi i a}, 1-s, 1-\frac{\log z}{2 \pi i}\right)\right\} \\
&(\operatorname{Re}(s)<0 ;|\arg (-\log (z) \bmod 2 \pi i)| \leq \pi \\
&\left.a \in \mathbb{C} \backslash \mathbb{Z}_{0}^{-}\right),
\end{aligned}
$$

which played a central role in obtaining expansion formulas.

The aim of this paper is to give some series representations of the Apostol-Bernoulli, Apostol-Euler, Apostol-Genocchi, and Apostol-Frobenius-Euler polynomials of higher order in terms of the generalized Hurwitz zeta function (1).

It is worthy to mention that throughout this work, the following convention is adopted for the binomial coefficient:

$$
\left(\begin{array}{ll}
n-1 \\
k-1
\end{array}\right):= \begin{cases}1, & n=k=1 \\
0, & n \geq 1, k=0 \\
\left(\begin{array}{l}
n-1 \\
k-1
\end{array}\right), & \text { otherwise. }\end{cases}
$$

\section{Expansion Formulas for $\Phi_{\mu}^{*}(z, s, a)$ and Fourier Series}

Few years ago, in order to give extensions of the works of Apostol [6] and Srivastava [4], Lin et al. [7] obtained an expansion formula for the generalized Hurwitz-Lerch zeta function $\Phi_{\mu}^{*}(z, s, a)$ defined by (9) in terms of the HurwitzLerch zeta function (3) by making use of the generalized Leibniz rule for fractional derivative [8, page 95, Equation (4.3)]. They proved the following expansion formula for the general Hurwitz-Lerch zeta function $\Phi_{\mu}^{*}(z, s, a)$ :

$$
\begin{aligned}
\Phi_{\mu}^{*}(z, s, a)= & i z^{-a} \Gamma(1-s) \sum_{j=0}^{\infty} \frac{(j-a+1)_{\mu-j-1}}{\Gamma(\mu-j) j !} \\
& \times \sum_{k=0}^{j}\left(\begin{array}{l}
j-1 \\
k-1
\end{array}\right)(1-s)_{k} B_{j-k}^{(j)}
\end{aligned}
$$




$$
\begin{aligned}
\cdot(2 \pi)^{s-k-1}[ & \exp \left(-\frac{1}{2}(s-k) \pi i\right) \\
& \times \Phi\left(e^{-2 \pi i a}, 1-s+k, \frac{\log z}{2 \pi i}\right) \\
& -\exp \left[\left(2 a+\frac{1}{2}(s-k)\right) \pi i\right] \\
& \left.\times \Phi\left(e^{2 \pi i a}, 1-s+k, 1-\frac{\log z}{2 \pi i}\right)\right],
\end{aligned}
$$

with $\operatorname{Re}(\mu)>0, s \in \mathbb{C},|\arg (-\log (z) \bmod 2 \pi i)| \leq \pi$, $a \in \mathbb{C} \backslash \mathbb{Z}_{0}^{-}$and where $B_{n}^{(\alpha)}:=B_{n}^{(\alpha)}(0)$ are the generalized Bernoulli numbers [9] given by the following generating function:

$$
\left(\frac{z}{e^{z}-1}\right)^{\alpha} e^{x z}=\sum_{n=0}^{\infty} B_{n}^{(\alpha)}(x) \frac{z^{n}}{n !} \quad\left(|z|<2 \pi ; 1^{\alpha}:=1\right) .
$$

From this expansion formula, many authors found several interesting explicit formulas for the Apostol-Bernoulli, Apostol-Euler, and Apostol-Genocchi polynomials at rational arguments [10-12]. For instance, Garg et al. [10, page 814, Equation (54)] obtained the following explicit formula for the Apostol-Bernoulli polynomials $\mathscr{B}_{n}^{(\alpha)}(x ; \lambda)$ (which will be defined in Section 3):

$$
\begin{aligned}
\mathscr{B}_{n}^{(l)}( & \left.\frac{p}{q} ; e^{2 \pi i \xi}\right) \\
= & \frac{i(-1)^{l} n !}{(l-1) !} \sum_{j=0}^{l-1}\left(\begin{array}{c}
l-1 \\
j
\end{array}\right)\left(j-\frac{p}{q}+1\right)_{l-j-1} \\
& \times \sum_{k=0}^{j}\left(\begin{array}{c}
j-1 \\
k-1
\end{array}\right)(1-l+n)_{k} \\
& \times B_{j-k}^{(j)}(2 \pi q)^{l-n-k-1} \\
& \cdot\left\{\sum_{r=1}^{q} \zeta\left(1-l+n+k, \frac{\xi+r-1}{q}\right)\right. \\
& \cdot \exp \left[-\left(\frac{1}{2}(l-n-k)+\frac{2(\xi+r-1) p}{q}\right) \pi i\right]
\end{aligned}
$$

$$
\begin{aligned}
& -\sum_{r=1}^{q} \zeta\left(1-l+n+k, \frac{r-\xi}{q}\right) \\
& \left.\cdot \exp \left[\left(\frac{1}{2}(l-n-k)+\frac{2(r-\xi) p}{q}\right) \pi i\right]\right\}
\end{aligned}
$$$$
(n, l \in \mathbb{N} ; \xi \in \mathbb{R} ; 0<\xi<1 ; p \in \mathbb{Z} ; q \in \mathbb{N}) .
$$

More recently, Gaboury and Bayad [13] applied a new generalized Leibniz rule for the fractional derivatives obtained by one of the authors [14] to obtain a new expansion formula involving the generalized Hurwitz-Lerch zeta function $\Phi_{\mu}^{*}(z, s, a)$. Explicitly, they proved the following theorem.

Theorem 1. The following expansion holds for $\Phi_{\mu}^{*}(z, s, a)$ :

$$
\begin{aligned}
& \Phi_{\mu}^{*}(z, s, a) \\
& =\frac{i \Gamma(1-s) z^{-a} \sin \beta \pi \sin (\beta+\mu-\omega) \pi}{\Gamma(\mu) \Gamma(1-\omega-a) \sin (\mu+\beta) \pi \sin (\beta-\omega) \pi} \\
& \cdot \sum_{m=0}^{\infty} \sum_{k=0}^{m} \sum_{j=0}^{k}\left(\begin{array}{c}
m-1 \\
k-1
\end{array}\right)\left(\begin{array}{l}
k \\
j
\end{array}\right) \\
& \times \frac{\Gamma(\mu-a-\omega-m)}{\Gamma(\mu-m) m !} B_{m-k}^{(m)} \\
& \times(2 \pi)^{s-1-j}(1-s)_{j} \\
& \cdot(\omega-1+m)_{k-j} \\
& \times\left[\exp \left(-\frac{(s-j) \pi i}{2}\right)\right. \\
& \times \Phi\left(e^{-2 \pi i a}, 1-s+j, \frac{\log z}{2 \pi i}\right) \\
& -\exp \left[\left(2 a+\frac{s-j}{2}\right) \pi i\right] \\
& \left.\times \Phi\left(e^{2 \pi i a}, 1-s+j, 1-\frac{\log z}{2 \pi i}\right)\right] \\
& \left(\mu \in \mathbb{C} \backslash \mathbb{Z}_{0}^{-} ; \omega \in \mathbb{C} ; \operatorname{Re}(1-\beta)>0 ; s \in \mathbb{C} ;\right. \\
& \left.|\arg (-\log (z) \bmod 2 \pi i)| \leq \pi ; a \in \mathbb{C} \backslash \mathbb{Z}_{0}^{-}\right) \text {. }
\end{aligned}
$$

More interestingly, setting

$$
z=e^{2 \pi i \xi}, \quad a=\frac{p}{q} \quad(p \in \mathbb{Z} ; q \in \mathbb{N} ; \xi \in \mathbb{R}),
$$

replacing $s$ by $\mu-s$, and then applying definition (12) and the series identity (14), we obtain the following corollary. 
Corollary 2. The following expansion holds true:

$$
\begin{aligned}
\Phi_{\mu}^{*}\left(e^{2 \pi i \xi}, \mu-s, \frac{p}{q}\right) \\
=\frac{i \Gamma(1-\mu+s) \sin \beta \pi \sin (\beta+\mu-\omega) \pi}{\Gamma(\mu) \Gamma(1-\omega-(p / q)) \sin (\mu+\beta) \pi \sin (\beta-\omega) \pi} \\
\times \sum_{m=0}^{\infty} \sum_{k=0}^{m} \sum_{j=0}^{k}\left(\begin{array}{c}
m-1 \\
k-1
\end{array}\right)\left(\begin{array}{c}
k \\
j
\end{array}\right) \\
\quad \cdot \frac{\Gamma(\mu-p / q-\omega-m)}{\Gamma(\mu-m) m !} \\
\quad \times B_{m-k}^{(m)}(2 \pi q)^{\mu-s-1-j} \\
\quad \times(1-\mu+s)_{j}(\omega-1+m)_{k-j} \\
.\left[\sum_{r=1}^{q} \zeta\left(1-\mu+s+j, \frac{\xi+r-1}{q}\right)\right. \\
\quad \times \exp \left[-i \pi\left(\frac{\mu-s-j}{2}+\frac{2(r-1+\xi) p}{q}\right)\right] \\
\quad-\sum_{r=1}^{q} \zeta\left(1-\mu+s+j, \frac{r-\xi}{q}\right) \\
\left.\quad \times \exp \left[i \pi\left(\frac{\mu-s-j}{2}+\frac{2(r-\xi) p}{q}\right)\right]\right] \\
s \in \mathbb{C} \backslash \mathbb{Z}-; \omega \in \mathbb{C} ; \operatorname{Re}(1-\beta)>0 ; \\
\left.s ; \xi<1 ; a \in \mathbb{C} \backslash \mathbb{Z}_{0}^{-}\right) .
\end{aligned}
$$

Remark 3. It is worthy to mention that (20) can be interpreted as the Fourier series expansion of $\Phi_{\mu}^{*}(z, s, a)$ with respect to the variable $a$. Moreover, if we set $\mu=1$ in (20), then, for $\operatorname{Re}(s)<0,0<|z|<1$ and $a \in[0,1]$, we find an expansion theorem due to Erdélyi et al. and Wirtinger [5, 15], namely,

$$
\Phi(z, s, a)=z^{-a} \Gamma(1-s) \sum_{n \in \mathbb{Z}} \frac{e^{2 \pi \text { ina }}}{(2 \pi i n-\log z)^{1-s}} .
$$

\section{Generalized Hurwitz-Lerch Zeta Function at Negative Integers}

In this section, we recall the definitions of the ApostolBernoulli, Apostol-Euler, Apostol-Genocchi, and ApostolFrobenius-Euler polynomials. Next, we give explicit relationships between these polynomials and the generalized Hurwitz-Lerch zeta function evaluated at negative integers.
Definition 4. The generalized Apostol-Bernoulli polynomials $\mathscr{B}_{n}^{(\alpha)}(x ; \lambda)$ are defined, for $\lambda, x \in \mathbb{C}$, by the following generating function $[16,17]$ :

$$
\begin{array}{r}
\left(\frac{t}{\lambda e^{t}-1}\right)^{\alpha} e^{x t}=\sum_{n=0}^{\infty} \mathscr{B}_{n}^{(\alpha)}(x ; \lambda) \frac{t^{n}}{n !}, \quad|t+\log (\lambda)|<2 \pi, \\
(\alpha \in \mathbb{C}, \text { if } \lambda \neq 1 ; \alpha \in \mathbb{N} \text { if } \lambda=1) .
\end{array}
$$

Definition 5. The generalized Apostol-Euler polynomials $\mathscr{E}_{n}^{(\alpha)}(x ; \lambda)$ are defined, for $\lambda, \alpha$ and $x \in \mathbb{C}$, by the following generating function [18]:

$$
\begin{array}{r}
\left(\frac{2}{\lambda e^{t}+1}\right)^{\alpha} e^{x t}=\sum_{n=0}^{\infty} \mathscr{E}_{n}^{(\alpha)}(x ; \lambda) \frac{t^{n}}{n !}, \quad|t+\log \lambda|<\pi, \\
(\alpha \in \mathbb{C}, \lambda \neq-1) .
\end{array}
$$

Definition 6. The generalized Apostol-Genocchi polynomials $\mathscr{G}_{n}^{(\alpha)}(x ; \lambda)$ are defined, for $\lambda, x \in \mathbb{C}$, by the following generating function $[17,19]$ :

$$
\left(\frac{2 t}{\lambda e^{t}+1}\right)^{\alpha} e^{x t}=\sum_{n=0}^{\infty} \mathscr{G}_{n}^{(\alpha)}(x ; \lambda) \frac{t^{n}}{n !}, \quad|t+\log (\lambda)|<\pi,
$$

$(\alpha \in \mathbb{C}$, if $\lambda \neq-1 ; \alpha \in \mathbb{N}$ if $\lambda=-1)$.

Definition 7. The generalized Apostol-Frobenius-Euler polynomials $\mathscr{H}_{n}^{(\alpha)}(x ; \lambda \mid u)$ are defined, for $\lambda, \alpha, u$ and $x \in \mathbb{C}$ with $u \neq 0,1$ and $\lambda \neq u$, by the following generating function [20]:

$$
\begin{gathered}
\left(\frac{1-u}{\lambda e^{t}-u}\right)^{\alpha} e^{x t}=\sum_{n=0}^{\infty} \mathscr{H}_{n}^{(\alpha)}(x ; \lambda \mid u) \frac{t^{n}}{n !}, \\
\left|t+\log \left(\frac{\lambda}{u}\right)\right|<\pi .
\end{gathered}
$$

Setting $\lambda=1$ and $\alpha=1$ in each of these definitions gives, respectively, the Bernoulli, Euler, Genocchi and FrobeniusEuler polynomials which polynomials have been largely investigated in the literature.

Recently, Bayad and Chikhi [21] established the following relationship between the Apostol-Euler polynomials $\mathscr{E}_{n}^{(\alpha)}(x ; \lambda)$ and the generalized Hurwitz-Lerch zeta function $\Phi_{\alpha}^{*}(z, s, a)$.

Theorem 8. Let $\lambda$ be a complex number such that $|\lambda| \leq 1$ and $\lambda \neq-1$. Let $\alpha$ and $x$ be two complex numbers such that $\operatorname{Re}(\alpha)>$ 0 and $\operatorname{Re}(x)>0$; then for all nonnegative integer $n$, one has

$$
\mathscr{E}_{n}^{(\alpha)}(x ; \lambda)=2^{\alpha} \Phi_{\alpha}^{*}(-\lambda,-n, x)
$$

From this last theorem, we can find the relationships between the generalized Hurwitz-Lerch zeta function $\Phi_{\alpha}^{*}(z, s, a)$ and the polynomials defined by (24), (26), and (27). By simple manipulations of their generating functions, 
we can rewrite each polynomials in function of the ApostolEuler polynomials. For instance, we have

$$
\left(\frac{1-u}{\lambda e^{t}-u}\right)^{\alpha} e^{x t}=\left(\frac{u-1}{2 u}\right)^{\alpha}\left(\frac{2}{(-\lambda / u) e^{t}+1}\right)^{\alpha} e^{x t}
$$

and thus,

$$
\mathscr{H}_{n}^{(\alpha)}(x ; \lambda \mid u)=\left(\frac{u-1}{2 u}\right)^{\alpha} \mathscr{E}_{n}^{(\alpha)}\left(x ; \frac{-\lambda}{u}\right)
$$

In a similar way, we can deduce the two next relationships between the Apostol-Bernoulli polynomials and the Apostol-Euler polynomials and between the ApostolGenocchi polynomials and the Apostol-Euler polynomials. Indeed, we have

$$
\begin{gathered}
\mathscr{B}_{n}^{(l)}(x ; \lambda)=l !\left(\begin{array}{c}
n \\
l
\end{array}\right)\left(\frac{-1}{2}\right)^{l} \mathscr{E}_{n-l}^{(l)}(x ;-\lambda) \quad\left(l \in \mathbb{N}_{0}, \lambda \neq 1\right), \\
\mathscr{G}_{n}^{(l)}(x ; \lambda)=l !\left(\begin{array}{c}
n \\
l
\end{array}\right) \mathscr{E}_{n-l}^{(l)}(x ; \lambda) \quad\left(l \in \mathbb{N}_{0}\right) .
\end{gathered}
$$

Now, with the help of Theorem 8, we obtain the following relations between each type of polynomials and the generalized Hurwitz-Lerch zeta function at negative integers:

$$
\begin{array}{r}
\mathscr{B}_{n}^{(l)}(x ; \lambda)=l !\left(\begin{array}{c}
n \\
l
\end{array}\right)(-1)^{l} \Phi_{l}^{*}(\lambda, l-n, x) \\
\left(|\lambda| \leq 1 ; \lambda \neq 1 ; l \in \mathbb{N}_{0}\right), \\
\mathscr{G}_{n}^{(l)}(x ; \lambda)=2^{l} l !\left(\begin{array}{c}
n \\
l
\end{array}\right) \Phi_{l}^{*}(-\lambda, l-n, x) \\
\left(|\lambda| \leq 1 ; \lambda \neq-1 ; l \in \mathbb{N}_{0}\right), \\
\mathscr{H}_{n}^{(\alpha)}(x ; \lambda \mid u)=\left(\frac{u-1}{u}\right)^{\alpha} \Phi_{\alpha}^{*}\left(\frac{\lambda}{u},-n, x\right) \\
\left(u \neq 0,1 ;\left|\frac{\lambda}{u}\right| \leq 1 ; \lambda \neq u\right) .
\end{array}
$$

\section{Explicit Representations for Several Polynomials at Rational Arguments}

This section is devoted to the presentation of the new series representations for each type of polynomials defined in Section 3. These representations are obtained by suitably combining the new expansion formula (20) and identities (28) and (32)-(34).
Theorem 9. The following explicit representation holds for the Apostol-Bernoulli polynomials at rational argument:

$$
\begin{aligned}
& \mathscr{B}_{n}^{(l)}\left(\frac{p}{q} ; e^{2 \pi i \xi}\right) \\
& =\frac{i(-1)^{l} n !}{\Gamma(l) \Gamma(1-\omega-(p / q))}
\end{aligned}
$$

$$
\begin{aligned}
& \times \sum_{m=0}^{l-1} \sum_{k=0}^{m} \sum_{j=0}^{k}\left(\begin{array}{c}
m-1 \\
k-1
\end{array}\right)\left(\begin{array}{c}
k \\
j
\end{array}\right) \\
& . \frac{\Gamma(l-(p / q)-\omega-m)}{\Gamma(l-m) m !} B_{m-k}^{(m)}(2 \pi q)^{l-n-1-j} \\
& \quad \times(1-l+n)_{j}(\omega-1+m)_{k-j} \\
& \cdot\left[\sum_{r=1}^{q} \zeta\left(1-l+n+j, \frac{\xi+r-1}{q}\right)\right. \\
& \quad \times \exp \left[-i \pi\left(\frac{l-n-j}{2}+\frac{2(r-1+\xi) p}{q}\right)\right] \\
& \quad-\sum_{r=1}^{q} \zeta\left(1-l+n+j, \frac{r-\xi}{q}\right) \\
& \left.\quad \times \exp \left[i \pi\left(\frac{l-n-j}{2}+\frac{2(r-\xi) p}{q}\right)\right]\right]
\end{aligned}
$$

$$
\begin{aligned}
& (l, n \in \mathbb{N} ; \omega \in \mathbb{C} ; \operatorname{Re}(1-\beta)>0 ; \xi \in \mathbb{R}, \\
& \left.0<\xi<1 ; \frac{p}{q} \in \mathbb{Q} \backslash \mathbb{Z}_{0}^{-}\right) .
\end{aligned}
$$

Proof. Setting $\mu=l, s=n$ with $n, l \in \mathbb{N}$ in the expansion formula (22) and with the help of the representation of Apostol-Bernoulli polynomials in terms of the generalized Hurwitz-Lerch zeta function (32), the result follows.

Theorem 10. The following explicit representation holds for the Apostol-Euler polynomials at rational argument:

$$
\begin{aligned}
\mathscr{E}_{n}^{(\alpha)}\left(\frac{p}{q} ; e^{2 \pi i \xi}\right) \\
=\frac{i \Gamma(1+n) 2^{\alpha} \sin \beta \pi \sin (\beta+\alpha-\omega) \pi}{\Gamma(\alpha) \Gamma(1-\omega-(p / q)) \sin (\alpha+\beta) \pi \sin (\beta-\omega) \pi} \\
\times \sum_{m=0}^{\infty} \sum_{k=0}^{m} \sum_{j=0}^{k}\left(\begin{array}{c}
k \\
j
\end{array}\right) \cdot\left(\begin{array}{c}
m-1 \\
k-1
\end{array}\right) \frac{\Gamma(\alpha-(p / q)-\omega-m)}{\Gamma(\alpha-m) m !} \\
\quad \times B_{m-k}^{(m)}(2 \pi q)^{-n-1-j}(1+n)_{j}(\omega-1+m)_{k-j} \\
\quad\left[\sum_{r=1}^{q} \zeta\left(1+n+j, \frac{2 \xi+2 r-1}{2 q}\right)\right. \\
\quad \times \exp \left[-i \pi\left(\frac{-n-j}{2}+\frac{(2 r+2 \xi-1) p}{q}\right)\right]
\end{aligned}
$$




$$
\begin{aligned}
& -\sum_{r=1}^{q} \zeta\left(1+n+j, \frac{2 r-2 \xi-1}{2 q}\right) \\
& \left.\quad \times \exp \left[i \pi\left(\frac{-n-j}{2}+\frac{(2 r-2 \xi-1) p}{q}\right)\right]\right] \\
& \left(\alpha \in \mathbb{C} \backslash \mathbb{Z}_{0}^{-} ; \omega \in \mathbb{C} ; \operatorname{Re}(1-\beta)>0 ;\right. \\
& \left.\xi \in \mathbb{R}, 0 \leq \xi \leq 1, \xi \neq \frac{1}{2} ; \frac{p}{q} \in \mathbb{Q} \backslash \mathbb{Z}_{0}^{-}\right) .
\end{aligned}
$$

Proof. Replacing $z$ by $-e^{2 \pi i \xi}(\xi \in \mathbb{R}), a$ by $(p / q)(p \in \mathbb{Z} ; q \in$ $\mathbb{N}), \mu$ by $\alpha(\alpha \in \mathbb{C}), s$ by $-n(n \in \mathbb{N})$ in (20) and using the identity (28) yield the result.

Theorem 11. The following explicit representation holds for the Apostol-Genocchi polynomials at rational argument:

$$
\begin{aligned}
& \mathscr{G}_{n}^{(l)}\left(\frac{p}{q} ; e^{2 \pi i \xi}\right) \\
& =\frac{i 2^{l} n !}{\Gamma(l) \Gamma(1-\omega-(p / q))} \\
& \times \sum_{m=0}^{l-1} \sum_{k=0}^{m} \sum_{j=0}^{k}\left(\begin{array}{l}
k \\
j
\end{array}\right)\left(\begin{array}{c}
m-1 \\
k-1
\end{array}\right) \\
& \cdot \frac{\Gamma(l-(p / q)-\omega-m)}{\Gamma(l-m) m !} B_{m-k}^{(m)}(2 \pi q)^{l-n-1-j} \\
& \times(1-l+n)_{j}(\omega-1+m)_{k-j} \\
& \cdot\left[\sum_{r=1}^{q} \zeta\left(1-l+n+j, \frac{2 \xi+2 r-1}{2 q}\right)\right. \\
& \times \exp \left[-i \pi\left(\frac{l-n-j}{2}+\frac{(2 r+2 \xi-1) p}{q}\right)\right] \\
& -\sum_{r=1}^{q} \zeta\left(1-l+n+j, \frac{2 r-2 \xi-1}{2 q}\right) \\
& \left.\times \exp \left[i \pi\left(\frac{l-n-j}{2}+\frac{(2 r-2 \xi-1) p}{q}\right)\right]\right] \\
& (l, n \in \mathbb{N} ; \omega \in \mathbb{C} ; \operatorname{Re}(1-\beta)>0 \\
& \left.\xi \in \mathbb{R}, 0 \leq \xi \leq 1, \xi \neq \frac{1}{2} ; \frac{p}{q} \in \mathbb{Q} \backslash \mathbb{Z}_{0}^{-}\right)
\end{aligned}
$$

Proof. Substituting $z$ by $-e^{2 \pi i \xi}(\xi \in \mathbb{R})$, $a$ by $(p / q)(p \in$ $\mathbb{Z} ; q \in \mathbb{N})$ and $s$ by $\mu-s(\mu \in \mathbb{C})$ in $(20)$ gives

$$
\begin{aligned}
& \Phi_{\mu}^{*}\left(-e^{2 \pi i \xi}, \mu-s, \frac{p}{q} ;\right) \\
& =\frac{i \Gamma(1-\mu+s) \sin \beta \pi \sin (\beta+\mu-\omega) \pi}{\Gamma(\mu) \Gamma(1-\omega-(p / q)) \sin (\mu+\beta) \pi \sin (\beta-\omega) \pi} \\
& \times \sum_{m=0}^{\infty} \sum_{k=0}^{m} \sum_{j=0}^{k} \cdot\left(\begin{array}{c}
k \\
j
\end{array}\right)\left(\begin{array}{c}
m-1 \\
k-1
\end{array}\right) \frac{\Gamma(\mu-(p / q)-\omega-m)}{\Gamma(\mu-m) m !} \\
& \times B_{m-k}^{(m)}(2 \pi q)^{\mu-s-1-j}(1-\mu+s)_{j}(\omega-1+m)_{k-j} \\
& \cdot\left[\sum_{r=1}^{q} \zeta\left(1-\mu+s+j, \frac{2 \xi+2 r-1}{2 q}\right)\right. \\
& \times \exp \left[-i \pi\left(\frac{\mu-s-j}{2}+\frac{(2 r+2 \xi-1) p}{q}\right)\right] \\
& -\sum_{r=1}^{q} \zeta\left(1-\mu-s+j, \frac{2 r-2 \xi-1}{2 q}\right) \\
& \left.\times \exp \left[i \pi\left(\frac{\mu-s-j}{2}+\frac{(2 r-2 \xi-1) p}{q}\right)\right]\right] .
\end{aligned}
$$

Putting $\mu=l(l \in \mathbb{N}), s=n(n \in \mathbb{N} ; n \geq l)$ and with the help of the identity (33), the result follows.

Theorem 12. The following explicit representation holds for the Apostol-Frobenius-Euler polynomials at rational argument:

$$
\begin{aligned}
& \mathscr{H}_{n}^{(\alpha)}\left(\frac{p}{q} ; e^{2 \pi i \xi} \mid e^{2 \pi i \theta}\right) \\
& =\frac{i\left(1-e^{-2 \pi i \theta}\right)^{\alpha} n ! \sin \beta \pi \sin (\beta+\alpha-\omega) \pi}{\Gamma(\alpha) \Gamma(1-\omega-(p / q)) \sin (\alpha+\beta) \pi \sin (\beta-\omega) \pi} \\
& \times \sum_{m=0}^{\infty} \sum_{k=0}^{m} \sum_{j=0}^{k} \cdot\left(\begin{array}{c}
m-1 \\
k-1
\end{array}\right)\left(\begin{array}{c}
k \\
j
\end{array}\right) \frac{\Gamma(\alpha-(p / q)-\omega-m)}{\Gamma(\alpha-m) m !} \\
& \times B_{m-k}^{(m)}(2 \pi q)^{-n-1-j}(1+n)_{j}(\omega-1+m)_{k-j} \\
& \quad\left[\begin{array}{l}
\sum_{r=1}^{q} \zeta\left(1+n+j, \frac{(\xi-\theta)+r-1}{q}\right) \\
\quad \times \exp \left[-i \pi\left(\frac{-n-j}{2}+\frac{2(r-1+(\xi-\theta)) p}{q}\right)\right]
\end{array}\right.
\end{aligned}
$$




$$
\begin{gathered}
-\sum_{r=1}^{q} \zeta\left(1+n+j, \frac{r-(\xi-\theta)}{q}\right) \\
\left.\times \exp \left[i \pi\left(\frac{-n-j}{2}+\frac{2(r-(\xi-\theta)) p}{q}\right)\right]\right] . \\
(\xi, \theta \in \mathbb{R}, 0 \leq \xi \leq 1,0<\theta<1, \\
0<|\xi-\theta|<1, \xi \neq \theta ; \frac{p}{q} \in \mathbb{Q} \backslash \mathbb{Z}_{0}^{-} ; \\
\left.\alpha \in \mathbb{C} \backslash \mathbb{Z}_{0}^{-} ; \omega \in \mathbb{C} ; \operatorname{Re}(1-\beta)>0\right) .
\end{gathered}
$$

Proof. The result follows by setting $z=e^{2 \pi i(\xi-\theta)}(\xi, \theta \in \mathbb{R})$, $a=(p / q)(p \in \mathbb{Z} ; q \in \mathbb{N}), \mu=\alpha(\alpha \in \mathbb{C}), s=-n(n \in \mathbb{N})$ in (20) and using the identity (34) with $\lambda=e^{2 \pi i(\xi)}$ and $u=$ $e^{2 \pi i(\theta)}$.

It is worthy to note that setting $l=1$ in (35) gives

$$
\begin{aligned}
\mathscr{B}_{n}\left(\frac{p}{q} ; e^{2 \pi i \xi}\right) & \\
=\frac{-n !}{(2 \pi q)^{n}}[ & \sum_{r=1}^{q} \zeta\left(n, \frac{\xi+r-1}{q}\right) \\
& \times \exp \left[-i \pi\left(\frac{-n}{2}+\frac{2(r-1+\xi) p}{q}\right)\right] \\
& -\sum_{r=1}^{q} \zeta\left(n, \frac{r-\xi}{q}\right) \\
& \left.\times \exp \left[i \pi\left(\frac{-n}{2}+\frac{2(r-\xi) p}{q}\right)\right]\right] \\
(n \in \mathbb{N} ; n \geq & \left.1 \xi \in \mathbb{R}, 0<\xi<1 ; \frac{p}{q} \in \mathbb{Q} \backslash \mathbb{Z}_{0}^{-}\right),
\end{aligned}
$$

a result given by Srivastava [4, page 84, Equation (4.6)].

Moreover, if we replace $\alpha$ by 1 in (36), we recover a result obtained by Luo [11, page 345, Equation (4.3)], namely,

$$
\begin{aligned}
& \mathscr{E}_{n}\left(\frac{p}{q} ; e^{2 \pi i \xi}\right) \\
&=\frac{2 n !}{(2 \pi q)^{n+1}}\left[\sum_{r=1}^{q} \zeta\left(1+n, \frac{2 \xi+2 r-1}{2 q}\right)\right. \\
& \\
& \quad \times \exp \left[-i \pi\left(\frac{-n-1}{2}+\frac{(2 r+2 \xi-1) p}{q}\right)\right]
\end{aligned}
$$

$$
\begin{aligned}
&-\sum_{r=1}^{q} \zeta\left(1+n, \frac{2 r-2 \xi-1}{2 q}\right) \\
&\left.\times \exp \left[i \pi\left(\frac{-n+1}{2}+\frac{(2 r-2 \xi-1) p}{q}\right)\right]\right] \\
&\left(\xi \in \mathbb{R}, 0 \leq \xi \leq 1, \xi \neq \frac{1}{2} ; p \in \mathbb{Z} ; q \in \mathbb{N} ; \frac{p}{q} \in \mathbb{Q} \backslash \mathbb{Z}_{0}^{-}\right) .
\end{aligned}
$$

\section{References}

[1] H. M. Srivastava and J. Choi, Series Associated with Zeta and Related Functions, Kluwer Academic Publishers, London, UK, 2001.

[2] S.-D. Lin and H. M. Srivastava, "Some families of the HurwitzLerch zeta functions and associated fractional derivative and other integral representations," Applied Mathematics and Computation, vol. 154, no. 3, pp. 725-733, 2004.

[3] S. P. Goyal and R. K. Laddha, "On the generalized Riemann zeta functions and the generalized Lambert transform," Ganita Sandesh, vol. 11, pp. 99-108, 1997.

[4] H. M. Srivastava, "Some formulas for the Bernoulli and Euler polynomials at rational arguments," Mathematical Proceedings of the Cambridge Philosophical Society, vol. 129, no. 1, pp. 77-84, 2000.

[5] A. Erdélyi, W. Magnus, F. Oberhettinger, and F. G. Tricomi, Higher Transcendental Functions, Volumes 1-3, McGraw-Hill Book, London, UK, 1953.

[6] T. M. Apostol, "On the Lerch zeta function," Pacific Journal of Mathematics, vol. 1, pp. 161-167, 1951.

[7] S.-D. Lin, H. M. Srivastava, and P.-Y. Wang, "Some expansion formulas for a class of generalized Hurwitz-Lerch zeta functions," Integral Transforms and Special Functions, vol. 17, no. 11, pp. 817-827, 2006.

[8] K. S. Miller and B. Ross, An Introduction of the Fractional Calculus and Fractional Differential Equations, John Wiley and Sons, New York, NY, USA, 1993.

[9] Y. Luke, The Special Functions and Their Approximations, vol. 12 of Mathematics in Science and Engineering, Academic Press, New York, NY, USA, 1969.

[10] M. Garg, K. Jain, and H. M. Srivastava, "Some relationships between the generalized Apostol-Bernoulli polynomials and Hurwitz-Lerch zeta functions," Integral Transforms and Special Functions, vol. 17, no. 11, pp. 803-815, 2006.

[11] Q.-M. Luo, "Some formulas for Apostol-Euler polynomials associated with Hurwitz zeta function at rational arguments," Applicable Analysis and Discrete Mathematics, vol. 3, no. 2, pp. 336-346, 2009.

[12] Q. Luo, "Extensions of the genocchi polynomials and their fourier expansions and integral representations," Osaka Journal of Mathematics, vol. 48, no. 2, pp. 291-310, 2011.

[13] S. Gaboury and A. Bayad, "Further expansion formulas for a class of generalized Hurwitz-Lerch zeta functions obtained by means of a new Leibniz rule for fractional derivatives," Advanced Studies in Contemporary Mathematics. In press.

[14] R. Tremblay, S. Gaboury, and B.-J. Fugère, "A new Leibniz rule and its integral analogue for fractional derivatives," Integral Transforms and Special Functions, vol. 24, no. 2, pp. 111-128, 2013. 
[15] W. Wirtinger, "Uber eine besondere Dirichletsche reihe," Journal für die Reine und Angewandte Mathematik, vol. 129, pp. 214219, 1905.

[16] Q.-M. Luo and H. M. Srivastava, "Some generalizations of the Apostol-Bernoulli and Apostol-Euler polynomials," Journal of Mathematical Analysis and Applications, vol. 308, no. 1, pp. 290302, 2005.

[17] H. M. Srivastava, B. Kurt, and Y. Simsek, "Some families of Genocchi type polynomials and their interpolation functions," Integral Transforms and Special Functions, vol. 23, no. 12, pp. 939-940, 2012.

[18] Q.-M. Luo, "Apostol-Euler polynomials of higher order and Gaussian hypergeometric functions," Taiwanese Journal of Mathematics, vol. 10, no. 4, pp. 917-925, 2006.

[19] Q.-M. Luo, " $q$-extensions for the Apostol-Genocchi polynomials," General Mathematics, vol. 17, no. 2, pp. 113-125, 2009.

[20] B. Kurt and Y. Simsek, "On the generalized Apostol-type Frobenius-Euler polynomials," Advances in Difference Equations, vol. 2013, article 1, 2013.

[21] A. Bayad and J. Chikhi, "Reduction and duality of the generalized Hurwitz-Lerch zetas," Fixed Point Theory and Applications, vol. 2013, article 82, 2013. 


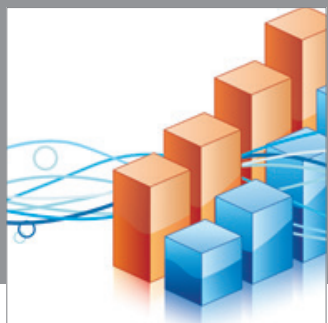

Advances in

Operations Research

mansans

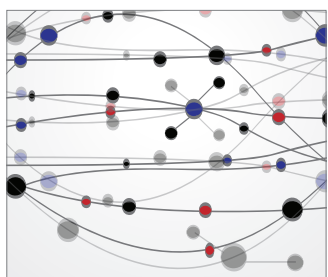

The Scientific World Journal
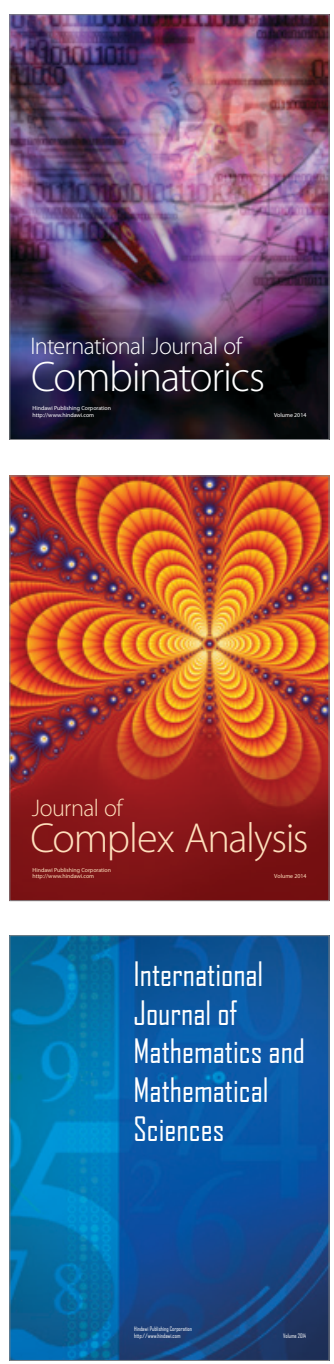
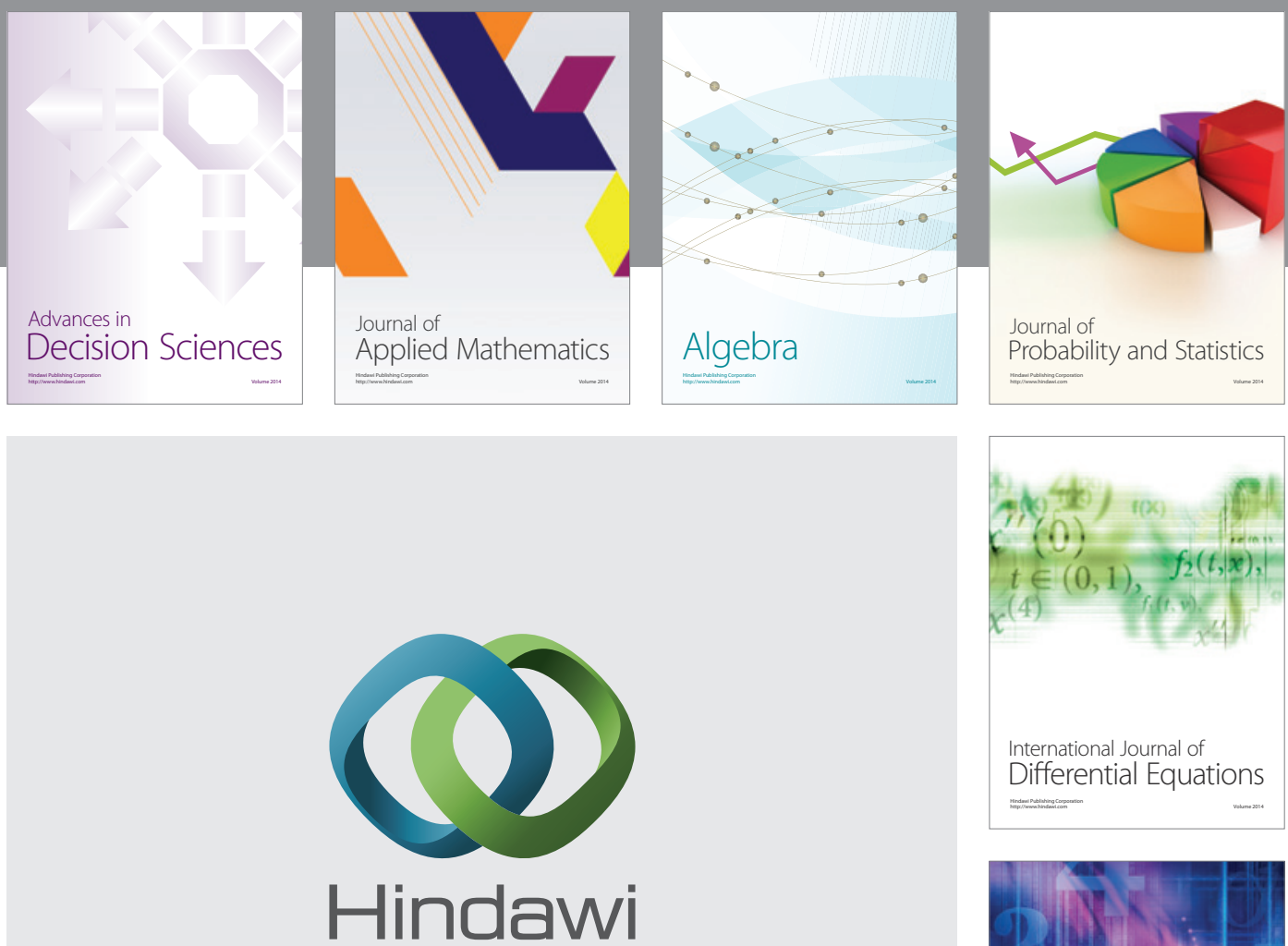

Submit your manuscripts at http://www.hindawi.com
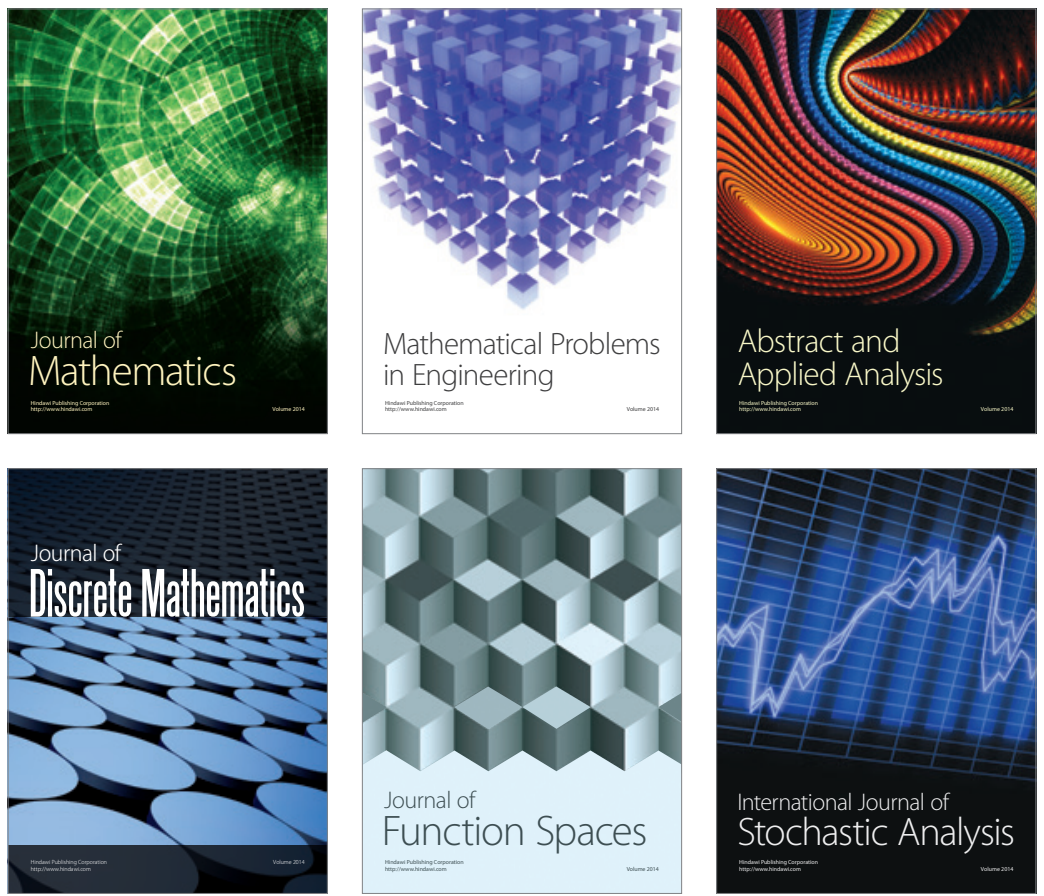

Journal of

Function Spaces

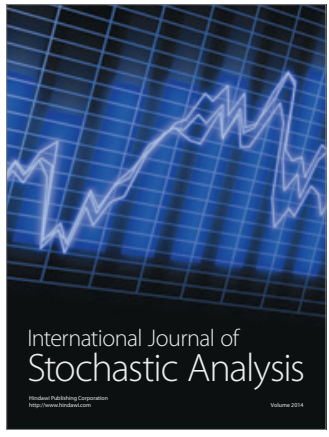

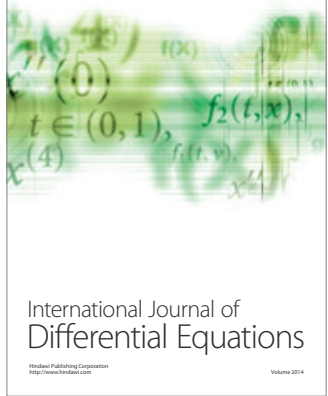
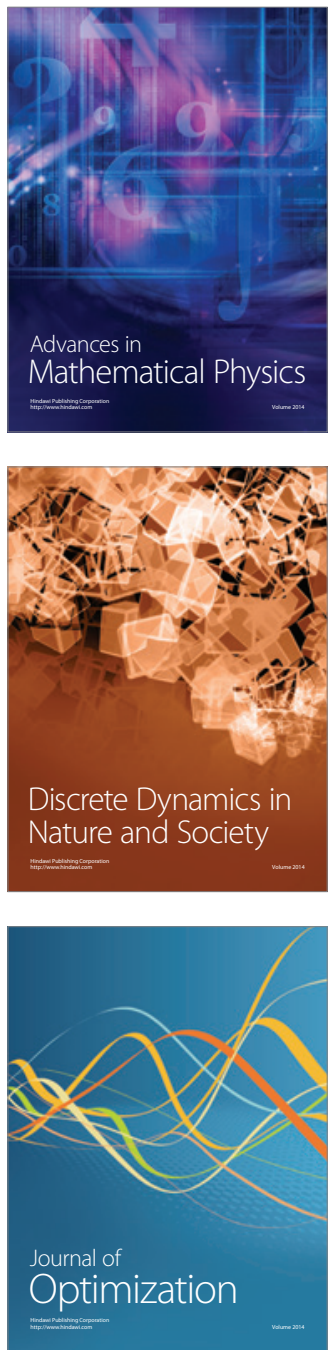